\title{
MiR-34a-5p/PD-L1 axis regulates cisplatin chemoresistance of ovarian cancer cells
}

\author{
Y. ZUO' ${ }^{1,2}$, W. ZHENG' ${ }^{2}$ J. LIU ${ }^{2}$, Q. TANG ${ }^{2}$, S. S. WANG ${ }^{2}$, X. S. YANG ${ }^{1, *}$
}

${ }^{1}$ Department of Obstetrics and Gynecology, Qilu Hospital of Shandong University, Jinan, Shandong 250012, China; ${ }^{2}$ Department of Gynecology, Affiliated Yantai Yuhuangding Hospital, Medical College of Qingdao University, Yantai, Shandong 264000, China

${ }^{*}$ Correspondence: xingshengyang@sdu.edu.cn

Received February 2, 2019 / Accepted June 26, 2019

\begin{abstract}
Ovarian cancer is the most lethal gynecologic malignancy in women with an increasing number of cases worldwide. Chemoresistance is the main obstacle for ovarian cancer treatment during clinical therapy. Previous studies found that programmed cell death 1 ligand 1 (PD-L1) was associated with chemoresistance of cancer. However, there were little reports about the function of PD-L1 involved in chemoresistance of ovarian cancer. In our study, cisplatin (DDP)-resistant SKOV3 and A2780 ovarian cancer cell lines (SKOV3/DDP and A2780/DDP) were established. We found that the expression of PD-L1 was increased and miR-34a-5p was decreased in DDP-resistant cells. PD-L1 silencing inhibited chemoresistance of DDP-resistant ovarian cancer cells to DDP, as evidenced by decreased proliferation, G1-phase cell cycle arrest and increased apoptosis. Western blot assay showed that in the presence of DDP, PD-L1 silencing decreased multidrug resistance protein 1 and Cyclin D1 protein levels, whereas increased cleaved-caspase-3 and cleaved-PARP protein levels in these cells. Moreover, we demonstrated that miR-34a-5p negatively regulated the expression of PD-L1 by targeting its 3'-untranslated region. The effects of miR-34a-5p mimic on DDP-treated SKOV3/DDP cells were reversed by the overexpression of PD-L1. Moreover, the tumorigenicity of DDP-resistant ovarian cancer cells in nude mice treated with DDP was attenuated by miR-34a-5p in vivo. The combined data indicate that miR-34a-5p/PD-L1 axis regulates DDP chemoresistance of ovarian cancer cells, providing a deeper insight into the treatment for ovarian cancer.
\end{abstract}

Key words: ovarian cancer, PD-L1, miR-34a-5p, DDP, chemoresistance

Ovarian cancer is the fourth leading cause of cancer deaths and considered as the most lethal gynecologic malignancy in women with an increasing number of cases worldwide [1-3]. Approximately 22,240 women were diagnosed with ovarian cancer and 14,070 died from the disease in 2018 [4]. Notably, about 75\% of patients present at FIGO (The International Federation of Gynecologists and Obstetricians) stages III or IV [5]. The 5-year survival rate is less than $10 \%$ in stage III-IV patients [6]. Therefore, the high degree of lethality is reflected in ovarian cancer. Currently, the screening and diagnostic methods for ovarian cancer are found, such as pelvic examination, transvaginal ultra-sound (TVU) and cancer antigen 125 (CA 125) as a tumor marker [7]. In addition, platinum- and taxane-based chemotherapy are used to follow the surgery. However, most patients experienced recurrence within 12-24 months and then died of progressively chemotherapy-resistant disease [8]. Therefore, it is important to perform the elimination of chemoresistance in ovarian cancer.
Programmed cell death 1 (PD-1), as a main cell-surface receptor of CD28 superfamily, is one of the major inhibitory molecules to reduce $\mathrm{T}$ cell activation $[9,10]$. It was frequently found to be expressed in activated T cells, whereas its major ligand, PD-L1, is typically expressed on antigen-presenting cells, but can be mainly found on tumor cells [11,12]. Reportedly, the PD-1 pathway was useful for maintaining self-tolerance and curbs $\mathrm{T}$ cells during an immune response, as well as preventing collateral damage to healthy tissues in healthy individuals [13]. However, cancers were found to elude immune surveillance via the PD-1/PD-L1 pathway, and high levels of expression of PD-L1 were related to poor prognosis of cancer in pre-clinical studies [14-16]. Similarly, there was a significantly poorer prognosis in patients with higher expression of PD-L1 than patients with lower expression in ovarian cancer [17]. In addition, carboplatin chemotherapy could induce the upregulation of PD-L1 in ovarian cancer [18]. Interestingly, the depletion of PD-L1 was reported to result in the significant reduction of cisplatin (DDP) resis- 
tance in non-small-cell lung cancer cells [19]. Nevertheless, the effects of PD-L1 on DDP resistance of ovarian cancer are still not reported.

MicroRNAs (miRNAs), well-known as small non-coding RNAs, can interact with the 3'-untranslated regions (3'-UTR) of multiple target messenger RNAs (mRNAs) and then regulate gene expression at the post-transcriptional level, including various tumors $[20,21]$. Notably, a recent report showed that miR-34a-5p was downregulated in human epithelial ovarian cancer [22]. In addition, miR-34a-5p could lead to enhancement of DDP sensitivity in ovarian cancer, medulloblastoma and muscle-invasive bladder cancer [23-25]. Interestingly, PD-L1 was demonstrated to be a target of miR-34a-5p in a few cancers, such as non-small cell lung cancer and acute myeloid leukemia $[26,27]$. Therefore, the data suggest that miR-34a-5p may be involved in regulating the chemosensitivity of ovarian cancer cells via targeting PD-L1.

In the current study, we indicated that miR-34a-5p could modulate chemosensitivity by targeting PD-L1 in ovarian cancer. The finding provides us the new therapeutic target for ovarian cancer.

\section{Materials and methods}

Cell culture, transfection and treatment. Human ovarian cancer cell lines (SKOV3 and A2780) and 293T cells were obtained from Procell Life Science and Technology Co. (Wuhan, China) and Shanghai Zhong Qiao Xin Zhou Biotechnology (Shanghai, China), respectively. All cells were cultured in DMEM (Gibco, Grand Island, NY, USA) containing $10 \%$ fetal bovine serum (HyClone, Logan, UT, USA) at $37^{\circ} \mathrm{C}$ under $5 \% \mathrm{CO}_{2}$. DDP was purchased from Dalian Meilun Biotech Co., (Dalian, China). Cells were transiently transfected with PD-L1 siRNA-1 (si1), PD-L1 si2, miR-34a-5p mimic, miR-34a-5p inhibitor, PD-L1 plasmid, or the corresponding negative controls for $24 \mathrm{~h}$ using Lipofectamine 2000 (Invitrogen, Carlsbad, USA). A2780/ DDP cells with stable overexpression of miR-34a-5p were established and used for in vivo experiment.

Cell proliferation assay. Cells $\left(5 \times 10^{3} /\right.$ well $)$ were seeded into 96-well plates. The transfected or non-transfected cells were incubated with serial dilutions of DDP $(0.625,1.25,2.5$, $5,10,20,40$ or $60 \mu \mathrm{M}$ ). After $72 \mathrm{~h}$, MTT solution (a terminal concentration of $0.5 \mathrm{mg} / \mathrm{ml}$ ) was added to each well for $5 \mathrm{~h}$. The medium was then removed and $150 \mu \mathrm{l}$ of DMSO (Beyotime, Shanghai, China) was added. Absorbance was assessed at $570 \mathrm{~nm}$. The half maximal inhibitory concentration (IC50) was determined. Cells were treated with DDP at the half of IC50 dose $24 \mathrm{~h}$ after transfection for following $24 \mathrm{~h}$ in experiments in vitro.

Western blot assay. Total protein was extracted from cells or tissues using RIPA buffer (Solarbio, Beijing, China) supplemented with PMSF (1 $\mathrm{mM})$ and quantified with BCA protein assay kit (Solarbio). Twenty $\mu \mathrm{g}$ of protein was separated with SDS-PAGE, transferred to PVDF membrane, and blocked using 5\% skim milk. Thereafter, the membranes were incubated with primary antibodies overnight at $4{ }^{\circ} \mathrm{C}$, including PD-L1 (1:500, Boster, Wuhan China), multidrug resistance protein 1 (MDR1) (1:1000, Bioss, Beijing China), Cyclin D1 (1:1000, Bioss), cleaved-caspase-3 (1:1000, CST, Danvers, MA, USA), cleaved-PARP (1:1000, CST) and GAPDH (1:10000, Proteintech, Wuhan China). Subsequently, the secondary antibodies IgG-HRP (1:5000, Solarbio) were used to incubate the membrane for $45 \mathrm{~min}$ at $37^{\circ} \mathrm{C}$. The bands were visualized by the enhanced chemiluminescence (Solarbio) and analyzed using Gel-Pro-Analyzer software.

Real-time qPCR. The total RNAs were extracted from cells or tissues using RNA extraction kit (Tiangen Biotech, Beijing, China) and reverse-transcribed to complementary DNA using M-MLV reverse transcriptase (TianGen Biotech). The PCR detection was performed with SYBR Green (Solarbio). The primer sequences of miR-34a-5p and PD-L1 were as follows: miR-34a-5p: forward primer, GGACTTGGCAGTGTCTTAGCTG; reverse primer, GTGCAGGGTCCGAGGTATTC; PD-L1: forward primer, AGAACTACCTCTGGCACAT; reverse primer, ATCCATCATTCTCCCTTT. U6 was the internal control of miR-34a-5p and GAPDH as the internal control of PD-L1. The relative expression of miR-34a-5p and PD-L1 was calculated using the $2^{-\Delta \Delta C t}$ method.

Cell cycle assay. The treated cells were collected and fixed with $70 \%$ ethanol at $4^{\circ} \mathrm{C}$ for $2 \mathrm{~h}$. The cells were then washed with PBS and stained with $25 \mu$ propidium iodide (PI) containing $10 \mu \mathrm{l}$ RNase A for $30 \mathrm{~min}$ in a dark environment at $37^{\circ} \mathrm{C}$. Finally, the cells in each cell-cycle phase (G1, $\mathrm{S}$, and G2) were assayed by flow cytometry (Aceabio, San Diego, USA).

Cell apoptosis assay. The treated cells were collected and incubated with $5 \mu \mathrm{l}$ Annexin V-FITC and $10 \mu \mathrm{l}$ PI for 10-20 min in dark environment at room temperature. Finally, the apoptotic percentage of cells was analyzed with flow cytometry (Aceabio).

Dual-luciferase reporter assay. The PD-L1 wild-type sequence (PD-L1-WT-3'UTR) containing the binding site of miR-34a-5p and the mutant sequence (PD-L1-MUT-3'UTR) were synthesized and cloned into the pmirGLO vector. Then 293T cells were co-transfected with pmirGLO-PD-L1-WT3'UTR or pmirGLO-PD-L1-MUT-3'UTR and NC mimic or miR-34a-5p mimic. Luciferase and Renilla activities were measured via the Dual-Luciferase Reporter Assay System (KeyGen, Nanjin, China). Luciferase activity was normalized to the Renilla luciferase activity.

Animal study. Twelve six-week-old male BABL/c-nu mice, weighing 18-20 g, were purchased from Beijing Huafukang Bioscience (Beijing, China) and housed in 12-hour light/dark cycles with free access to food and water. This study was approved by the Institutional Animal Care and Use Committee of the Qilu Hospital of Shandong University and 
carried out according to the Guidelines for the Care and Use of Laboratory Animals.

Mice were divided into two groups ( $\mathrm{N}=6$ per group). One group was subcutaneously inoculated with $2 \times 10^{6} \mathrm{miR}-34 \mathrm{a}-$ 5p-overexpressing A2780/DDP cells. Another group was inoculated with $2 \times 10^{6} \mathrm{miR}-\mathrm{NC}$-overexpressing A2780/DDP cells. DDP (10 mg/kg, twice a week) was intraperitoneally injected into mice following the tumor volume reached about $100 \mathrm{~mm}^{3}$. Tumor volume was measured every second day for three weeks. Afterward, tumor tissues were isolated, photographed and stored in liquid nitrogen for real-time PCR and western blot analysis.

Statistical analysis. GraphPad Prism 7.0 software was used to perform statistical analyses. The data were presented as mean \pm SD. The two-tailed non-paired Student's t-test was used to compare differences between two group. For multiple comparisons, one-way or two-way ANOVA followed by Tukey's test was used. p-values of less than 0.05 were considered statistically significant.

\section{Results}

PD-L1 and miR-34a-5p levels in DDP-resistant ovarian cancer cells. In order to demonstrate that DDP-resistant SKOV3 and A2780 (SKOV3/DDP, A2780/DDP) cell lines were established, we first used the MTT assay to access the effects of serial dilutions of DDP on cell proliferation in SKOV3, SKOV3/DDP, A2780 and A2780/DDP cells. Cell viability was lower in DDP-resistant cells with the increase of DDP concentration, compared with the parental cells (Figure 1A). In addition, there was an increase of the DDP IC50 value in DDP-resistant cells (Figure 1A). Subsequently,
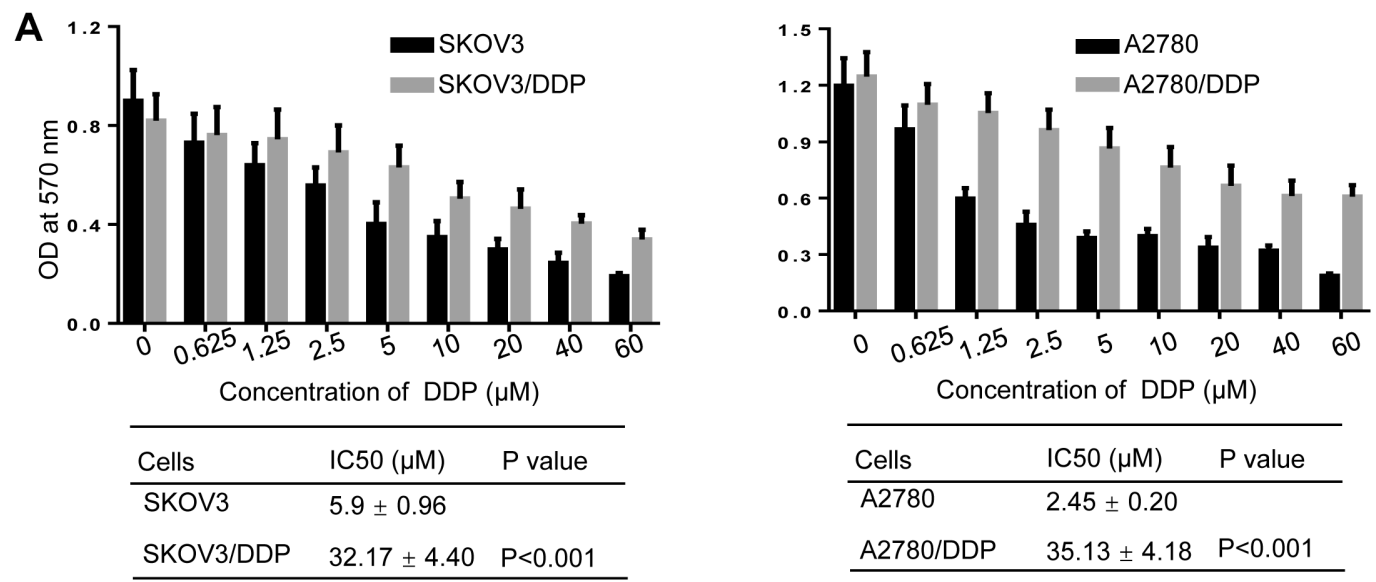

B

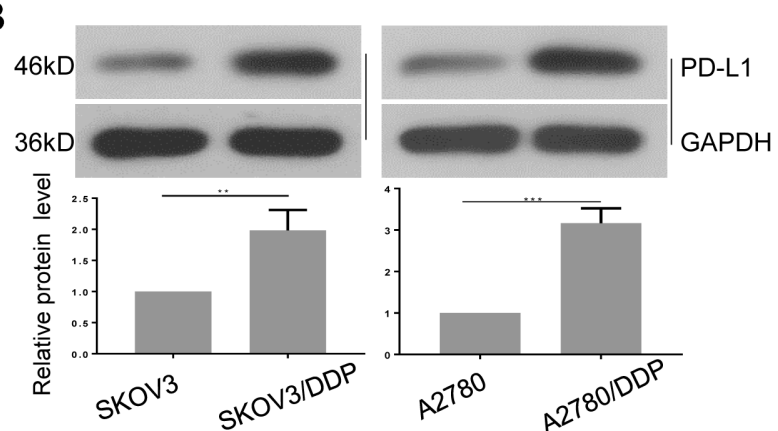

C
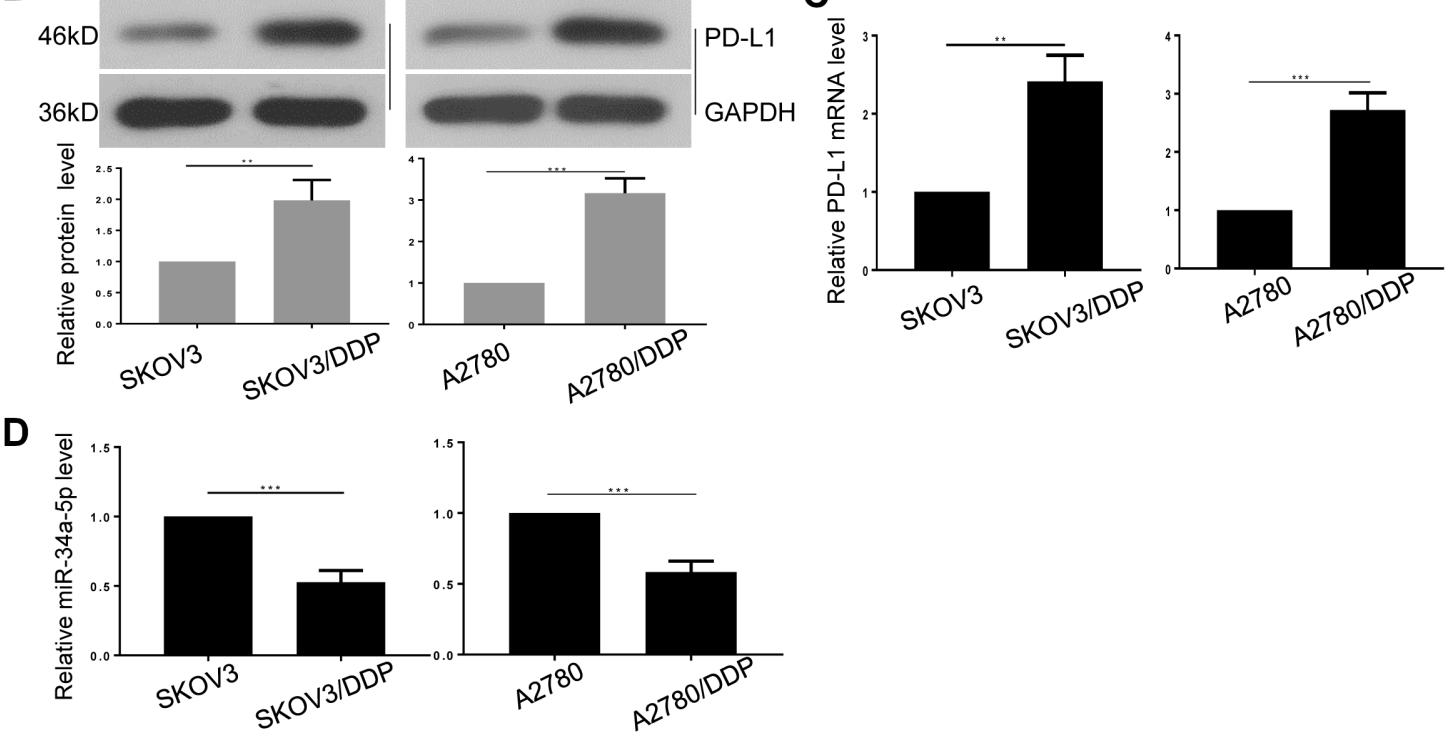

Figure 1. PD-L1 and miR-34a-5p levels in DDP-resistant ovarian cancer cells. A) The effect of the gradient concentration of DDP on cell viability in DDP-resistant ovarian cancer cells (SKOV3/DDP and A2780/DDP) and wild-type cells (SKOV3 and A2780) was measured by MTT assay. The IC50 values of DDP for each cell line were calculated and presented below. B and C) Western blot and real-time PCR were employed to detect PD-L1 protein and mRNA levels. D) The expression levels of miR-34a-5p were determined by real-time $P C R . n=3$ experiments, ${ }^{\star *}$ indicated $p<0.01,{ }^{* * *}$ indicated $p<0.001$. 
western blot assay and real-time PCR detected increased PD-L1 level (Figures $1 \mathrm{~B}$ and 1C), whereas decreased miR-34a-5p level was measured using real-time PCR in DDP-resistant cells (Figure 1D).

The effect of DDP on cell properties in PD-L1-silenced DDP-resistant cells. Given that PD-L1 was increased in DDP-resistant cells, the effect of DDP on cell properties was evaluated following PD-L1 knockdown. Western blot assay indicated that PD-L1 was successfully downregulated in SKOV3/DDP (Figure 2A). Furthermore, the serial dilutions of DDP were used to treat the PD-L1-silenced DDP-resistant cells and the inhibition of cell proliferation was shown with MTT assay (Figure 2B). The IC50 value of DDP was decreased in DDP-resistant cells with downregulation of PD-L1 (Figure 2B). Moreover, flow cytometry assay demonstrated that DDP treatment at the half of IC50 concentra- tion led to the accumulated percentage of cells in G1 phase and decreased the percentage of cells in the S and G2 phases (Figure 2C) and the increased apoptosis (Figure 2D) in the PD-L1-silenced SKOV3/DDP and A2780/DDP cells. PD-L1 silencing in DDP-treated DDP-resistant ovarian carcinoma cells led to a significant change in cell morphology characteristic for apoptosis (Supplementary Figure S1). Besides, western blot assay revealed that DDP administration caused the downregulation of MDR1 and Cyclin D1 protein levels, whereas resulted in the increase of cleaved-caspase- 3 and cleaved-PARP protein levels in PD-L1-silenced DDP-resistant cells (Figure 2E).

The relationship between miR-34a-5p and PD-L1 in ovarian cancer cells. To explore whether miR-34a-5p could regulate the PD-L1 expression in ovarian cancer, we first conducted miR-34a-5p overexpression and knockdown.
A
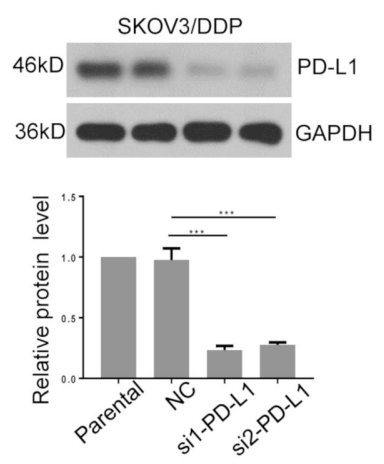

B

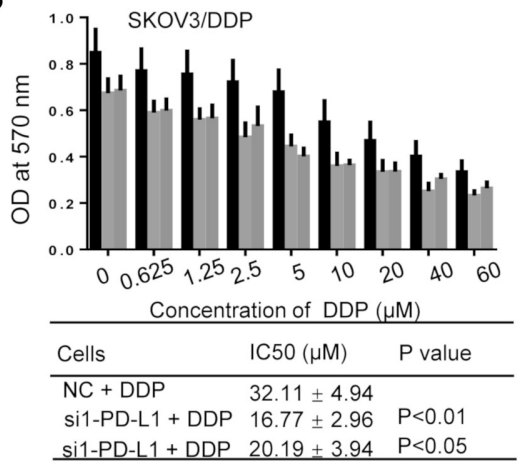

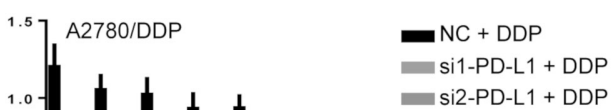

si2-PD-L1+ DDP

C
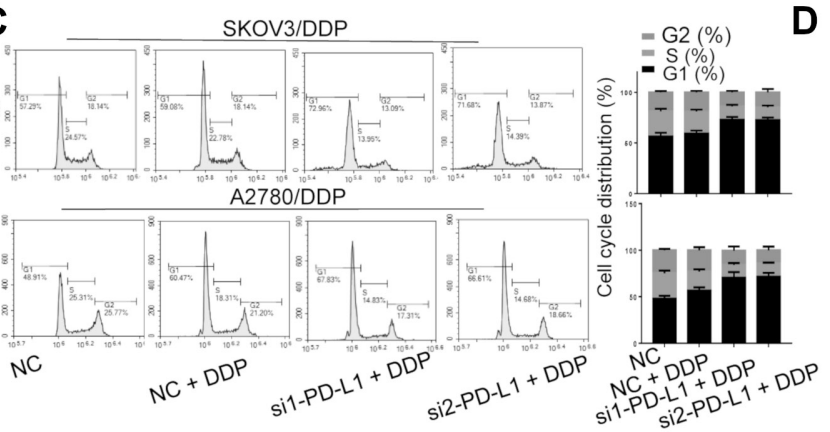

D
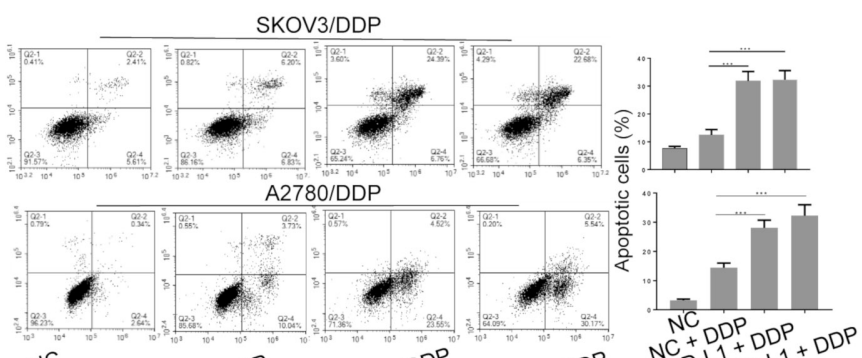

E
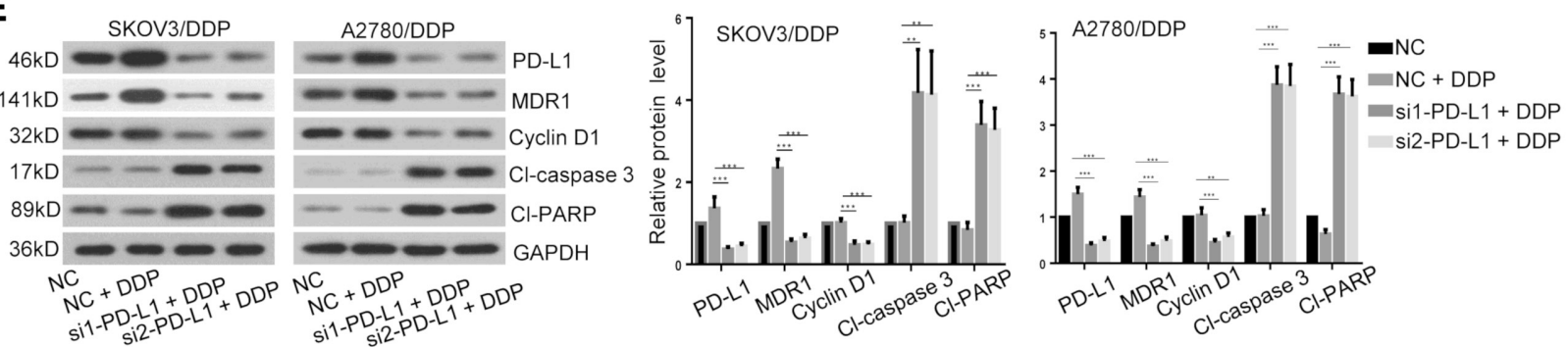

Figure 2. The effect of PD-L1 knockdown on DDP-resistant ovarian cancer cells treated with DDP. A) The interference efficiency of two siRNAs (sil and si2) for PD-L1 in SKOV3/DDP was measured by western blotting. B) The influence of PD-L1 silencing on proliferation in DDP-resistant ovarian cancer cells treated with a gradient concentration of DDP was detected using MTT assay. The IC50 values of DDP for each cell line were calculated and presented below. $\mathrm{C}$ and D) The effect of PD-L1 downregulation on cell cycle phase distribution and cell apoptosis in DDP-resistant ovarian cancer cells treated with DDP was analyzed via flow cytometry. E) Protein levels of PD-L1, MDR1, Cyclin D1, cleaved caspase 3, and cleaved PARP were assayed through western blotting. $\mathrm{n}=3$ experiments, ${ }^{* *}$ indicated $\mathrm{p}<0.01,{ }^{* *}$ indicated $\mathrm{p}<0.001$. 
The results from real-time PCR showed that miR-34a-5p overexpression or knockdown was successfully performed in SKOV3/DDP and A2780/DDP cells or SKOV3 and A2780 cells, respectively (Figures $3 \mathrm{~A}$ and 3D). Furthermore, real-time PCR and western blot assay demonstrated that miR-34a-5p mimic decreased PD-L1 mRNA and protein levels (Figures 3B and 3C), whereas miR-34a-5p inhibitor increased PD-L1 mRNA and protein levels (Figures 3E and $3 F)$. Importantly, a dual luciferase reporter assay confirmed that PD-L1 was a target gene of miR-34a-5p (Figure 3G).

The effect of PD-L1 on miR-34a-5p overexpressing SKOV3/DDP cells treated with DDP. After a gradient concentration of DDP treatment, MTT assay revealed that PD-L1 overexpression reversed the inhibition of proliferation induced by miR-34a-5p in SKOV3/DDP cells (Figure 4A). The IC50 value of DDP caused by miR-34a-5p was inhibited by the overexpression of PD-L1 (Figure 4A). In addition, the arrested G1 phase and increased apoptosis mediated by $\mathrm{miR}-34 \mathrm{a}-5 \mathrm{p}$ were reversed by $\mathrm{PD}-\mathrm{L} 1$ overexpression (Figures $4 \mathrm{~B}$ and $4 \mathrm{C}$ ). Furthermore, PD-L1 overexpression also reversed the expression change of MDR1, Cyclin D1, cleaved-caspase- 3 and cleaved-PARP caused by miR-34a-5p in SKOV3/DDP cells (Figure 4D).

The effect of miR-34a-5p on tumorigenesis of A2780/ DDP cells in nude mice with DDP treatment. Moreover, the influence of miR-34a-5p on tumorigenesis of A2780/ DDP was investigated in nude mice treated with DDP. As shown in Figures 5A and 5B, tumor growth was significantly inhibited by DDP in mice injected with miR-34a-5p overexpressing A2780/DDP cells compared with control mice. The expression of miR-34a-5p in tumor tissues was confirmed by real-time PCR (Figure 5C). Western blot assay showed that
A
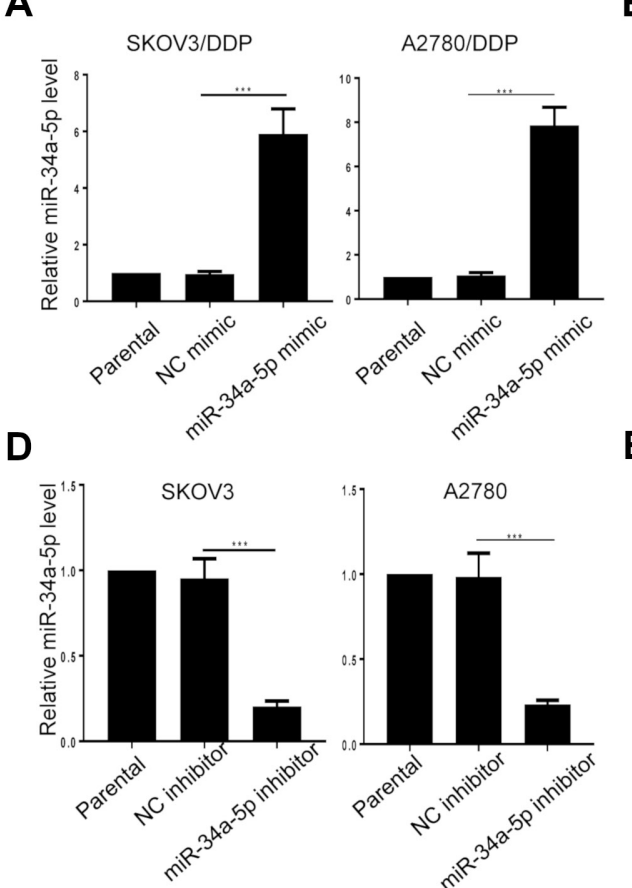

B

E

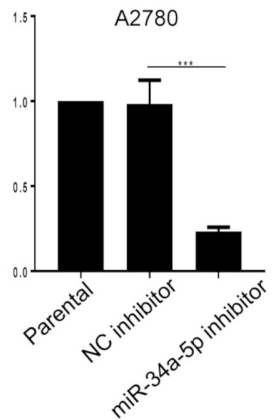

B C
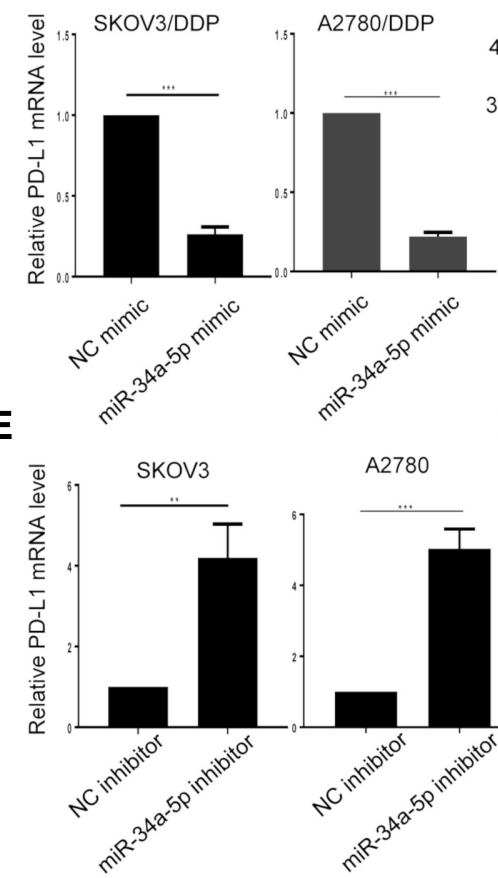

C
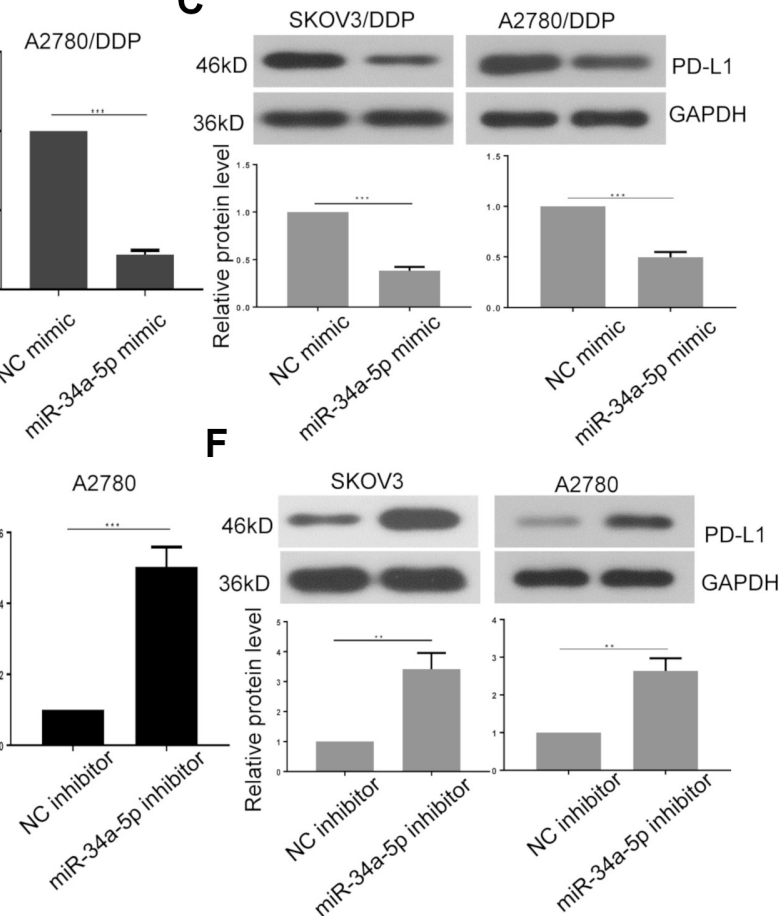

G

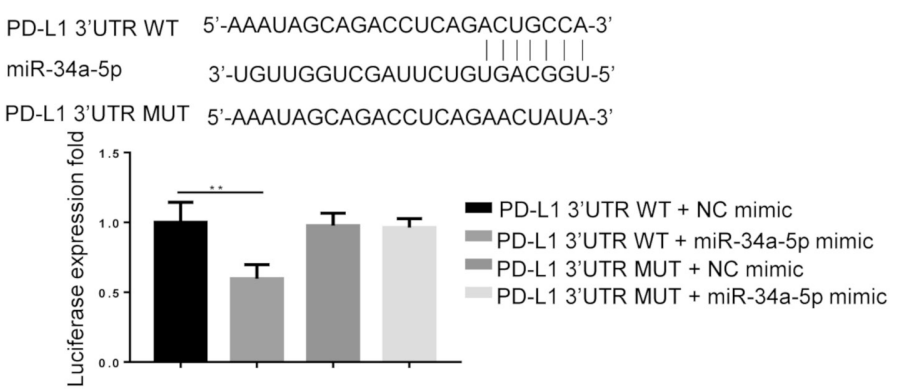

Figure 3. The relationship between miR-34a-5p and PD-L1 in ovarian cancer cells. A) The efficiency of miR-34a-5p mimic transfection was validated in SKOV3/DDP and A2780/DDP cells by real-time PCR. B and C) The effect of miR-34a-5p overexpression on PD-L1 expression was assessed by real-time PCR and western blot analyses. D) The efficiency of miR-34a-5p inhibitor transfection was confirmed in SKOV3 and A2780 cells by real-time PCR. E and F) The effect of miR-34a-5p knockdown on PD-L1 expression was determined by real-time PCR and western blot analyses. G) The luciferase reporter gene assay was employed to confirm the binding of miR-34a-5p with PD-L1. $\mathrm{n}=3$ experiments, ${ }^{\star *}$ indicated $\mathrm{p}<0.01,{ }^{* *}$ indicated $\mathrm{p}<0.001$. 


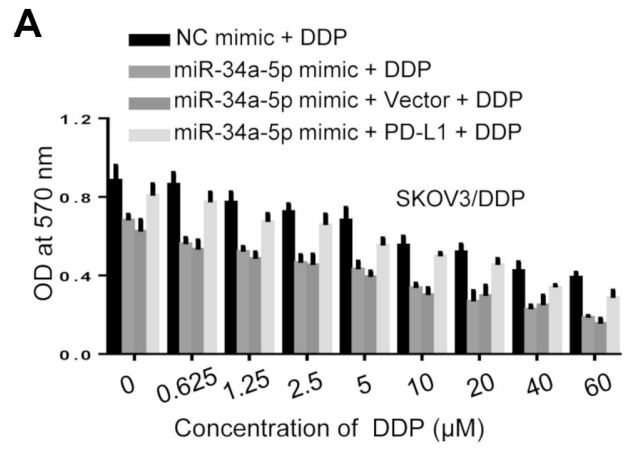

\begin{tabular}{lll}
\hline \multicolumn{1}{c}{ SKOV3/DDP } & IC50 $(\mu \mathrm{M})$ & P value \\
\hline NC mimic + DDP & $29.09 \pm 2.58$ & \\
miR-34a-5p mimic + DDP & $10.63 \pm 1.82$ & $P<0.001$ \\
miR-34a-5p mimic + Vector + DDP & $12.66 \pm 1.43$ & \\
miR-34a-5p mimic + PD-L1 + DDP & $22.66 \pm 2.70$ & $P<0.01$ \\
\hline
\end{tabular}

B

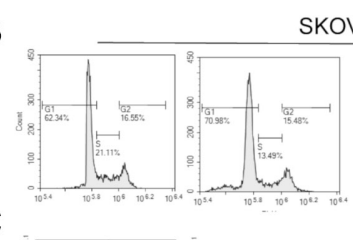

SKOV3/DDP
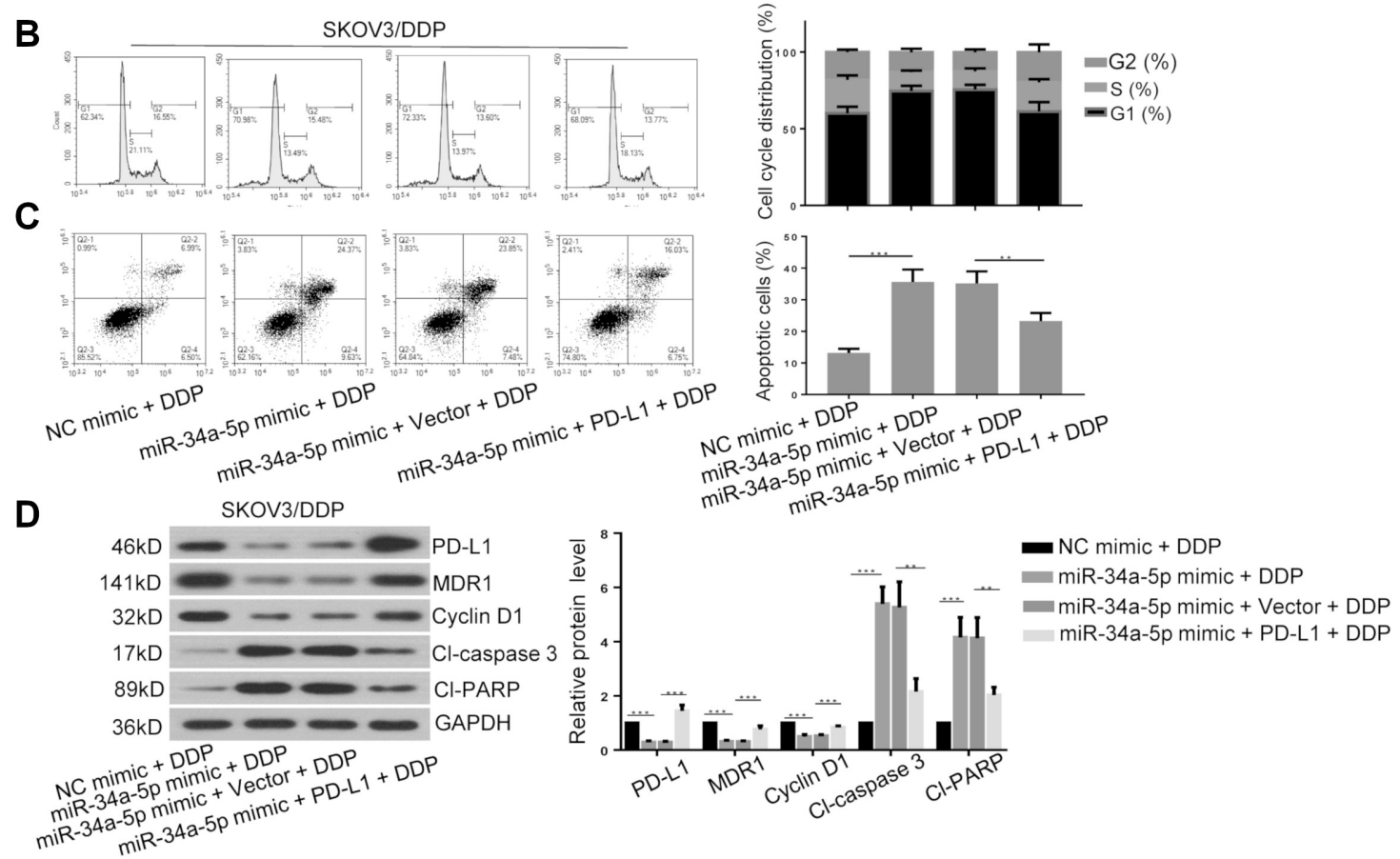

Figure 4. The effect of PD-L1 on miR-34a-5p-overexpressing SKOV3/DDP cells with DDP treatment. A) The effect of a gradient concentration of DDP on proliferation in SKOV3/DDP cells co-transfected with miR-34a-5p mimic and PD-L1 plasmid was measured by MTT assay. The IC50 values of DDP for each cell line were calculated and presented right. B and C) The effect of DDP on cell cycle phase distribution and cell apoptosis in SKOV3/DDP cells co-transfected with miR-34a-5p mimic and PD-L1 plasmid was analyzed via flow cytometry. D) Western blot assay was used to determine the protein levels of PD-L1, MDR1, Cyclin D1, cleaved caspase 3, and cleaved PARP. $n=3$ experiments, ${ }^{* *}$ indicated p $<0.01,{ }^{* * *}$ indicated $\mathrm{p}<0.001$.

the expression levels of PD-L1, MDR1 and Cyclin D1 were decreased, and the expression levels of cleaved-caspase-3 and cleaved-PARP were increased in miR-34a-5p-upregulated tumor tissues following DDP treatment (Figure 5D). Lastly, a schematic diagram was drawn to illustrate the regulation of miR-34a-5p/PD-L1 axis in DDP-resistant ovarian carcinoma cells (Figure 6).

\section{Discussion}

In the present study, DDP-resistant SKOV3 and A2780 cell lines were established. The increased PD-L1 and decreased miR-34a-5p were measured in DDP-resistant cells. Knockdown of PD-L1 expression inhibited chemore- sistance of DDP-resistant cells to DDP. Moreover, we demonstrated that PD-L1 is a target gene of miR-34a-5p in ovarian cancer. The effects of miR-34a-5p mimic on SKOV3/DDP treated with DDP could be reversed by the overexpression of PD-L1. Finally, we demonstrated that the tumorigenicity of DDP-resistant ovarian cancer cells in nude mice treated with DDP was inhibited by miR-34a-5p. Together, this study indicated that miR-34a-5p/PD-L1 axis could play an important role in DDP chemoresistance in ovarian cancer.

Early reports have shown that PD-L1 level was increased in breast cancer and melanoma cells with cytotoxic drug treatment $[28,29]$. In addition, the reduced miR-34a level was related to chemotherapy resistance in bladder cancer, lung cancer and Ewing's sarcoma [25, 30-32]. However, 
A

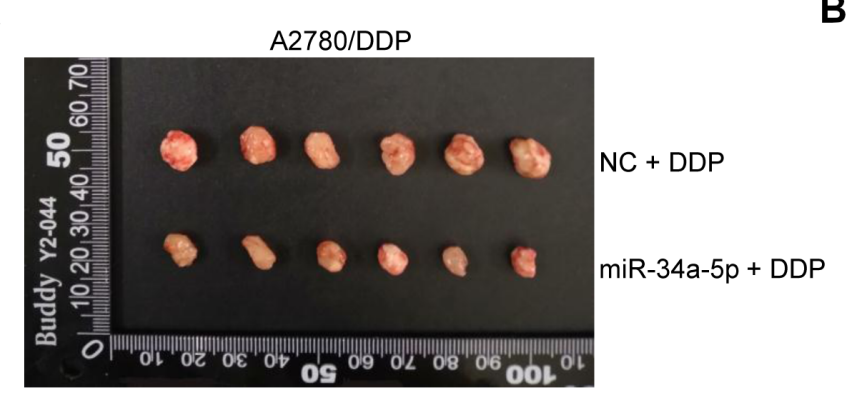

C

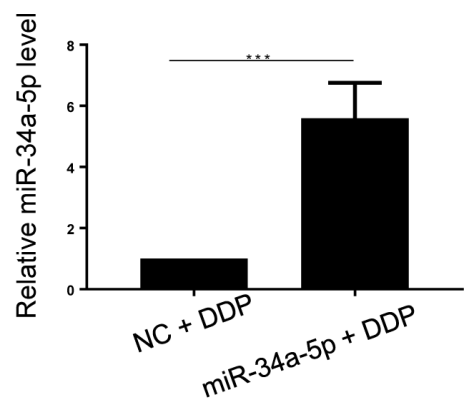

B

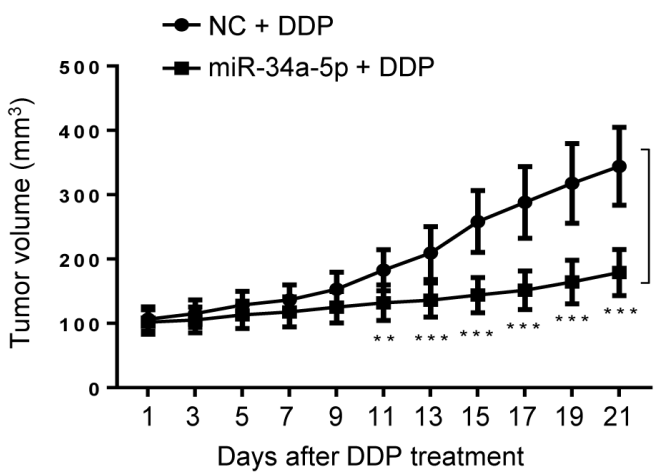

D
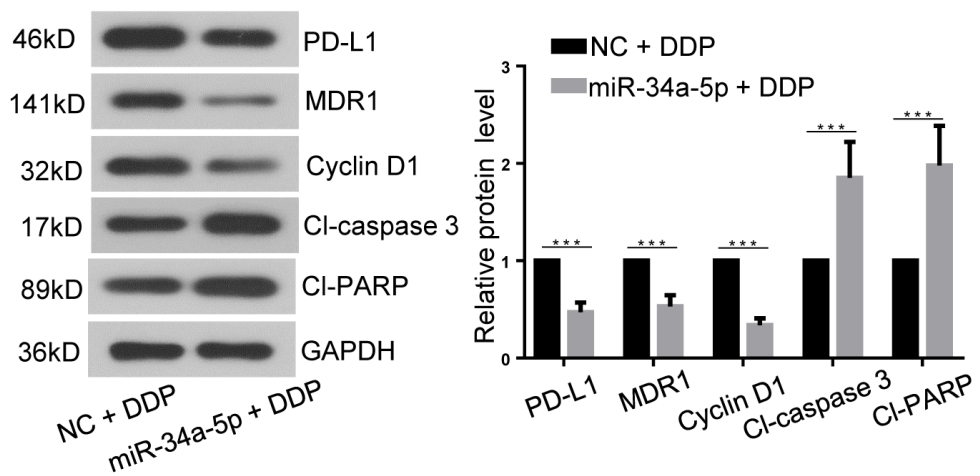

Figure 5. Overexpression of miR-34a-5p inhibited tumorigenicity of A2780/DDP cells in nude mice treated with DDP. A) Images of tumor tissues from nude mice injected with miR-34a-5p-overexpressing A2780/DDP cells or control cells and treated with DDP. B) The change of tumor volume following administration of DDP in nude mice inoculated with miR-34a-5p-overexpressing A2780/DDP cells or control cells. C) The expression levels of miR-34a-5p in tumor tissues were analyzed by real-time PCR. D) Western blot assay was applied to assess the expression of PD-L1, MDR1, Cyclin D1, cleaved caspase 3 , and cleaved PARP in tumor tissues. $n=6$ mice/group, ${ }^{\star *}$ indicated $\mathrm{p}<0.01,{ }^{* * *}$ indicated $\mathrm{p}<0.001$.

another study showed that miR-34a-5p could facilitate the multi-chemoresistance of osteosarcoma [33]. In our study, we found the enhanced PD-L1 level and decreased miR-34a-5p level in SKOV3/DDP and A2780/DDP cells. PD-L1 was induced by proto-oncogene $\mathrm{Bcl} 3$ and involved in the proliferation of ovarian cancer cells [34]. Thyroxine suppressed resveratrol-mediated apoptosis by $\mathrm{PD}-\mathrm{L} 1$ in ovarian cancer cells [35]. Here, we demonstrated that PD-L1 silencing inhibited chemoresistance of DDP-resistant ovarian cancer cells to DDP, as evidenced by the inhibition of cell proliferation, the arrest of the cell cycle at G1 phase, and the increase of apoptotic cells. Meanwhile, the expression levels of MDR1 and Cyclin D1 were decreased, and the expression levels of cleaved-caspase- 3 and cleaved-PARP were increased in DDP-treated drug-resistant cells following knockdown of PD-L1. The data suggested that PD-L1 knockdown could alleviate the chemoresistance of ovarian cancer cells to DDP.

Previous studies have indicated that PD-L1 was a target gene of miR-34a-5p [26, 27]. We deduce that miR-34a-5p may play a role in chemoresistance of ovarian cancer cells by targeting PD-L1. As expected, the expression of PD-L1 was negatively regulated by miR-34a-5p in DDP-resistant ovarian cancer cells. Moreover, upregulation of miR-34a-5p enhanced chemosensitivity of DDP-resistant ovarian cancer

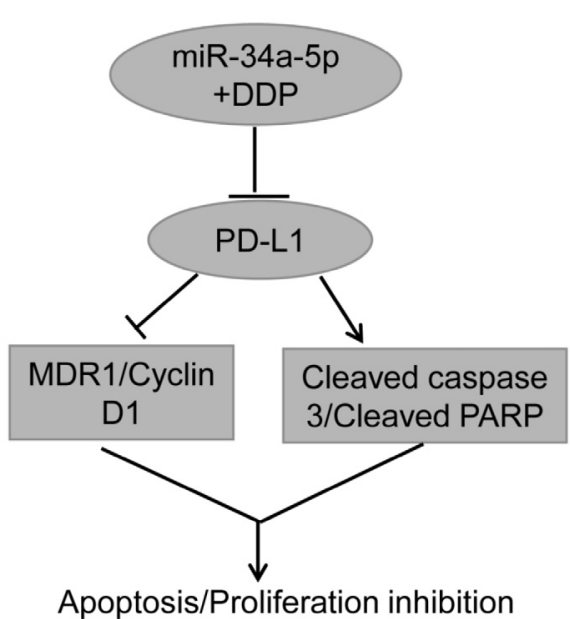

Figure 6. A schematic diagram of miR-34a-5p regulated PD-L1 in DDPresistant ovarian cancer cells.

cells to DDP. Importantly, PD-L1 overexpression reversed the effects of miR-34a-5p on DDP-treated drug-resistant cells, indicating that miR-34a-5p inhibited chemoresistance of ovarian cancer cells by targeting PD-L1. One of the limitations of this study is the limited number of cell lines included. 
Moreover, further clinical studies are necessary to determine the role of miR-34a-5p/PD-L1 axis in DDP-resistant ovarian cancer.

In conclusion, this study demonstrates that miR-34a-5p/ PD-L1 axis exerts a key role in chemoresistance of ovarian cancer, indicating a promising therapeutic approach for treating DDP-resistant ovarian cancer.

Supplementary information is available in the online version of the paper.

\section{References}

[1] KARST AM, DRAPKIN R. Ovarian cancer pathogenesis: a model in evolution. J Oncol 2010; 2010: 932371. https://doi. org/10.1155/2010/932371

[2] SIEGEL RL, MILLER KD, JEMAL A. Cancer Statistics, 2017. CA Cancer J Clin 2017; 67: 7-30. https://doi.org/10.3322/ caac. 21387

[3] WEBB PM, JORDAN SJ. Epidemiology of epithelial ovarian cancer. Best Pract Res Clin Obstet Gynaecol 2017; 41: 3-14. https://doi.org/10.1016/j.bpobgyn.2016.08.006

[4] SIEGEL RL, MILLER KD, JEMAL A. Cancer statistics, 2018. CA Cancer J Clin 2018; 68: 7-30. https://doi.org/10.3322/ caac. 21442

[5] HEINTZ AP, ODICINO F, MAISONNEUVE P, QUINN MA, BENEDET JL, et al. Carcinoma of the ovary. FIGO 26th Annual Report on the Results of Treatment in Gynecological Cancer. Int J Gynaecol Obstet 2006; 95 Suppl 1: S161-192. https://doi.org/10.1016/S0020-7292(06)60033-7

[6] DUFFY MJ, BONFRER JM, KULPA J, RUSTIN GJ, SOLETORMOS G et al. CA125 in ovarian cancer: European Group on Tumor Markers guidelines for clinical use. Int J Gynecol Cancer 2005; 15: 679-691. https://doi.org/10.1111/j.15251438.2005.00130.x

[7] SMITH RA, ANDREWS KS, BROOKS D, FEDEWA SA, MANASSARAM-BAPTISTE D et al. Cancer screening in the United States, 2018: A review of current American Cancer Society guidelines and current issues in cancer screening. CA Cancer J Clin 2018; 68: 297-316. https://doi.org/10.3322/ caac. 21446

[8] AU YEUNG CL, CO NN, TSURUGA T, YEUNG TL, KWAN SY et al. Exosomal transfer of stroma-derived miR21 confers paclitaxel resistance in ovarian cancer cells through targeting APAF1. Nat Commun 2016; 7: 11150. https://doi. org/10.1038/ncomms 11150

[9] CHO H, KANG H, LEE HH, KIM CW. Programmed Cell Death 1 (PD-1) and Cytotoxic T Lymphocyte-Associated Antigen 4 (CTLA-4) in Viral Hepatitis. Int J Mol Sci 2017; 18. https://doi.org/10.3390/ijms18071517

[10] KEIR ME, FRANCISCO LM, SHARPE AH. PD-1 and its ligands in T-cell immunity. Curr Opin Immunol 2007; 19: 309-314. https://doi.org/10.1016/j.coi.2007.04.012

[11] OKAZAKI T, CHIKUMA S, IWAI Y, FAGARASAN S, HONJO T. A rheostat for immune responses: the unique properties of PD-1 and their advantages for clinical application. Nat Immunol 2013; 14: 1212-1218. https://doi.org/10.1038/ni.2762
[12] ISHIDA Y, AGATA Y, SHIBAHARA K, HONJO T. Induced expression of PD-1, a novel member of the immunoglobulin gene superfamily, upon programmed cell death. EMBO J 1992; 11: 3887-3895. https://doi.org/10.1002/j.1460-2075.1992. tb05481.x

[13] KEIR ME, BUTTE MJ, FREEMAN GJ, SHARPE AH. PD-1 and its ligands in tolerance and immunity. Annu Rev Immunol 2008; 26: 677-704. https://doi.org/10.1146/annurev.immunol.26.021607.090331

[14] HINO R, KABASHIMA K, KATO Y, YAGI H, NAKAMURA $\mathrm{M}$ et al. Tumor cell expression of programmed cell death-1 ligand 1 is a prognostic factor for malignant melanoma. Cancer 2010; 116: 1757-1766. https://doi.org/10.1002/ cncr.24899

[15] THOMPSON RH, GILLETT MD, CHEVILLE JC, LOHSE $\mathrm{CM}, \mathrm{DONG} \mathrm{H}$ et al. Costimulatory B7-H1 in renal cell carcinoma patients: Indicator of tumor aggressiveness and potential therapeutic target. Proc Natl Acad Sci U S A 2004; 101: 17174-17179. https://doi.org/10.1073/pnas.0406351101

[16] NAKANISHI J, WADA Y, MATSUMOTO K, AZUMA M, KIKUCHI K et al. Overexpression of B7-H1 (PD-L1) significantly associates with tumor grade and postoperative prognosis in human urothelial cancers. Cancer Immunol Immunother 2007; 56: 1173-1182. https://doi.org/10.1007/ s00262-006-0266-z

[17] HAMANISHI J, MANDAI M, IWASAKI M, OKAZAKI T, TANAKA Y et al. Programmed cell death 1 ligand 1 and tumor-infiltrating CD8+ T lymphocytes are prognostic factors of human ovarian cancer. Proc Natl Acad Sci U S A 2007; 104: 3360-3365. https://doi.org/10.1073/pnas.0611533104

[18] PENG J, HAMANISHI J, MATSUMURA N, ABIKO K, MURAT K et al. Chemotherapy Induces Programmed Cell Death-Ligand 1 Overexpression via the Nuclear Factor-kappaB to Foster an Immunosuppressive Tumor Microenvironment in Ovarian Cancer. Cancer Res 2015; 75: 5034-5045. https://doi.org/10.1158/0008-5472.CAN-14-3098

[19] ZHANG P, MA Y, LV C, HUANG M, LI M et al. Upregulation of programmed cell death ligand 1 promotes resistance response in non-small-cell lung cancer patients treated with neo-adjuvant chemotherapy. Cancer Sci 2016; 107: $1563-$ 1571. https://doi.org/10.1111/cas. 13072

[20] PILLAI RS. MicroRNA function: multiple mechanisms for a tiny RNA? RNA 2005; 11: 1753-1761. https://doi. org/10.1261/rna.2248605

[21] IORIO MV, VISONE R, DI LEVA G, DONATI V, PETROCCA $F$ et al. MicroRNA signatures in human ovarian cancer. Cancer Res 2007; 67: 8699-8707. https://doi. org/10.1158/0008-5472.CAN-07-1936

[22] CORNEY DC, HWANG CI, MATOSO A, VOGT M, FLESKEN-NIKITIN A et al. Frequent downregulation of miR-34 family in human ovarian cancers. Clin Cancer Res 2010; 16: 1119-1128. https://doi.org/10.1158/1078-0432. CCR-09-2642

[23] LV T, SONG K, ZHANG L, LI W, CHEN Y et al. miRNA34a decreases ovarian cancer cell proliferation and chemoresistance by targeting HDAC1. Biochem Cell Biol 2018; 96: 663-671. https://doi.org/10.1139/bcb-2018-0031 
[24] WEERARATNE SD, AMANI V, NEISS A, TEIDER N, SCOTT DK et al. miR-34a confers chemosensitivity through modulation of MAGE-A and p53 in medulloblastoma. Neuro Oncol 2011; 13: 165-175. https://doi.org/10.1093/neuonc/noq179

[25] VINALL RL, RIPOLL AZ, WANG S, PAN CX, DEVERE WHITE RW. MiR-34a chemosensitizes bladder cancer cells to cisplatin treatment regardless of $\mathrm{p} 53-\mathrm{Rb}$ pathway status. Int J Cancer 2012; 130: 2526-2538. https://doi.org/10.1002/ ijc. 26256

[26] CORTEZ MA, IVAN C, VALDECANAS D, WANG X, PELTIER HJ et al. PDL1 Regulation by p53 via miR-34. J Natl Cancer Inst 2015; 108. https://doi.org/10.1093/jnci/djv303

[27] PYZER AR, STROOPINSKY D, ROSENBLATT J, ANASTASIADOU E, RAJABI $\mathrm{H}$ et al. MUC1 inhibition leads to decrease in PD-L1 levels via upregulation of miRNAs. Leukemia 2017; 31: 2780-2790. https://doi.org/10.1038/ leu. 2017.163

[28] GHEBEH H, LEHE C, BARHOUSH E, AL-ROMAIH K, TULBAH A et al. Doxorubicin downregulates cell surface B7-H1 expression and upregulates its nuclear expression in breast cancer cells: role of $\mathrm{B} 7-\mathrm{H} 1$ as an anti-apoptotic molecule. Breast Cancer Res 2010; 12: R48. https://doi. org/10.1186/bcr2605

[29] JIANG X, ZHOU J, GIOBBIE-HURDER A, WARGO J, HODI FS. The activation of MAPK in melanoma cells resistant to BRAF inhibition promotes PD-L1 expression that is reversible by MEK and PI3K inhibition. Clin Cancer Res 2013; 19: 598-609. https://doi.org/10.1158/1078-0432.CCR$12-2731$
[30] WIGGINS JF, RUFFINO L, KELNAR K, OMOTOLA M, PATRAWALA L et al. Development of a lung cancer therapeutic based on the tumor suppressor microRNA-34. Cancer Res 2010; 70: 5923-5930. https://doi.org/10.1158/0008-5472. CAN-10-0655

[31] JI X, WANG Z, GEAMANU A, GOJA A, SARKAR FH et al. Delta-tocotrienol suppresses Notch-1 pathway by upregulating miR-34a in nonsmall cell lung cancer cells. Int J Cancer 2012; 131: 2668-2677. https://doi.org/10.1002/ijc.27549

[32] NAKATANI F, FERRACIN M, MANARA MC, VENTURA S, DEL MONACO V et al. miR-34a predicts survival of Ewing's sarcoma patients and directly influences cell chemosensitivity and malignancy. J Pathol 2012; 226: 796-805. https://doi.org/10.1002/path.3007

[33] PU Y, ZHAO F, LI Y, CUI M, WANG $\mathrm{H}$ et al. The miR-34a$5 \mathrm{p}$ promotes the multi-chemoresistance of osteosarcoma via repression of the AGTR1 gene. BMC Cancer 2017; 17: 45. https://doi.org/10.1186/s12885-016-3002-X

[34] ZOU Y, UDDIN MM, PADMANABHAN S, ZHU Y, BU P et al. The proto-oncogene $\mathrm{Bcl} 3$ induces immune checkpoint PD-L1 expression, mediating proliferation of ovarian cancer cells. J Biol Chem 2018; 293: 15483-15496. https://doi. org/10.1074/jbc.RA118.004084

[35] CHIN YT, WEI PL, HO Y, NANA AW, CHANGOU CA, et al. Thyroxine inhibits resveratrol-caused apoptosis by PD-L1 in ovarian cancer cells. Endocr Relat Cancer 2018; 25: 533545. https://doi.org/10.1530/ERC-17-0376 


\section{MiR-34a-5p/PD-L1 axis regulates cisplatin chemoresistance of ovarian cancer cells}

Y. ZUO', ${ }^{1,2}$, W. ZHENG ${ }^{2}$, J. LIU ${ }^{2}$, Q. TANG ${ }^{2}$, S. S. WANG ${ }^{2}$, X. S. YANG ${ }^{1, *}$

Supplementary Information

SKOV3/DDP
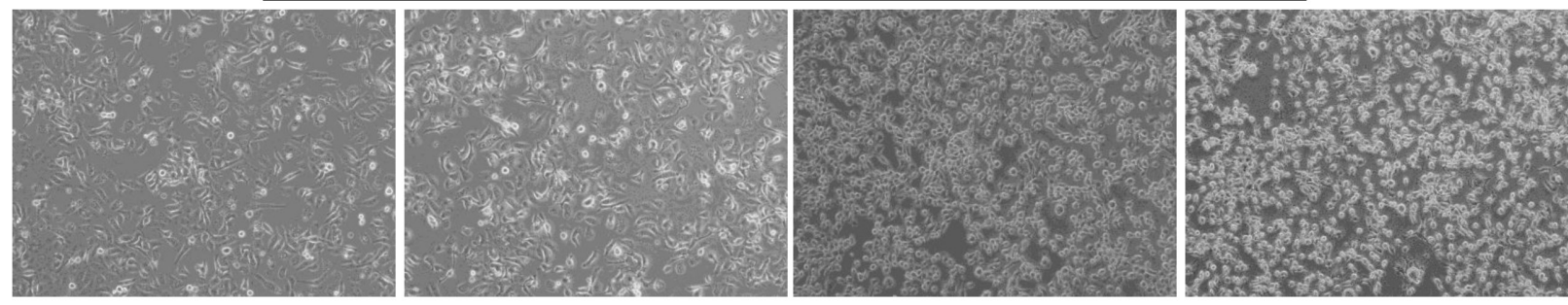

A2780/DDP

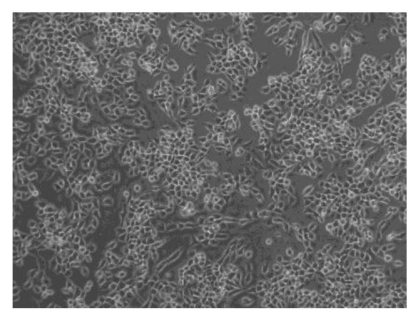

NC

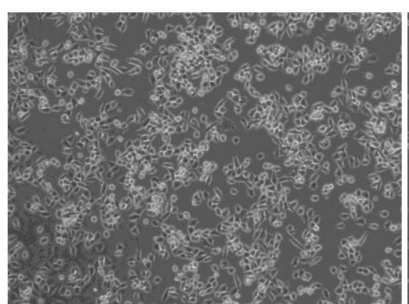

$N C+D D P$

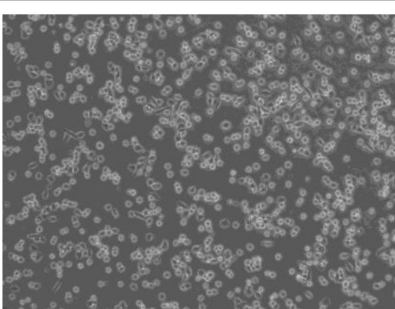

si1-PD-L1 + DDP

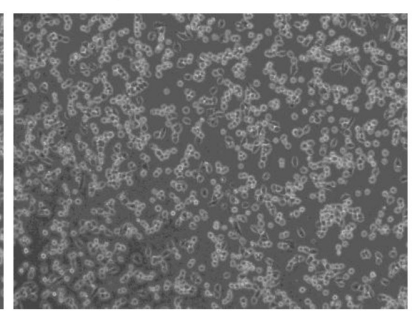

si2-PD-L1 + DDP

Supplementary Figure S1. The effect of PD-L1 knockdown on cell morphology in SKOV3/DDP and A2780/DDP cells treated with DDP. 